\title{
A Lie algebroid framework for non-holonomic systems
}

\author{
Tom Mestdag and Bavo Langerock ${ }^{1}$ \\ Department of Mathematical Physics and Astronomy, Ghent University, Krijgslaan 281, \\ B-9000 Ghent, Belgium \\ E-mail: Tom.Mestdag@UGent.be and Bavo.Langerock@UGent.be
}

Received 21 October 2004, in final form 1 December 2004

Published 19 January 2005

Online at stacks.iop.org/JPhysA/38/1097

\begin{abstract}
In order to obtain a framework in which both non-holonomic mechanical systems and non-holonomic mechanical systems with symmetry can be described, we introduce in this paper the notion of a Lagrangian system on a subbundle of a Lie algebroid.
\end{abstract}

PACS numbers: $02.40 .-\mathrm{k}, 45.20 . \mathrm{Jj}$

Mathematics Subject Classification: 17B66, 53C05, 70G45, 70H03, $70 \mathrm{H} 05$

\section{Introduction}

It is well known that the geometric description of the Euler-Lagrange equations of a mechanical system, derived from Hamilton's principle, heavily relies on the intrinsic geometry of the tangent bundle $T Q$ of the configuration space $Q$. In the case that the Lagrangian $L$ is invariant under the action of a Lie group $G$, Hamilton's principle can be reformulated into a reduced variational principle that takes into account the symmetry properties of the system. The advantage is that the reduced variational principle leads to equations defined on a reduced space, i.e. equations depending on 'fewer independent variables'. Thus, instead of considering the Euler-Lagrange equations on the total space $Q$, we are interested in the reduced equations, the so-called Lagrange-Poincaré ${ }^{2}$ equations, which are formulated on the Atiyah quotient bundle $T Q / G \rightarrow Q / G$ (see e.g. [13]). It is clear that the geometry of such quotient bundles now becomes of interest. Weinstein [24] has pushed ahead our understanding in this matter by showing that the geometric structure which lies at the heart of the Lagrange-Poincaré equations is essentially the same as the one of the Euler-Lagrange equations, namely that of a Lie algebroid. Therefore, the geometry of 'a Lagrangian system on a Lie algebroid' unifies the geometry of both standard Lagrangian systems and those where the symmetry properties are taking into account. In the case of standard Lagrangian systems, the tangent bundle carries a canonical Lie algebroid structure which is given by the usual Lie algebra of vector fields on $Q$.

1 Postdoctoral Fellow of the Fund for Scientific Research-Flanders (Belgium).

2 We use the terminology of $[6,7]$. 
For systems with symmetry, we make use of the so-called Atiyah algebroid structure on $T Q / G \rightarrow Q / G$ to describe the evolution equations (see e.g. [6, 9]).

In this paper, we mainly deal with mechanical systems that are subject to some kinematical constraints (i.e. depending on the velocity), also called non-holonomic constraints. The governing equations for these models are the so-called Lagrange-d'Alembert equations. For systems having additional symmetry properties, the equations of motion can be reduced to the Lagrange-d'Alembert-Poincaré equations (a fairly complete list of references on nonholonomic mechanics can be found in $[2,8]$ ). The main purpose of this paper is to show that the Lie algebroid structure constitutes a unifying geometric structure for describing simultaneously both types of Lagrangian systems with non-holonomic constraints. Throughout this paper, we will develop the basic geometric concepts and objects that are involved.

We believe that at this stage it is instructive to provide a local version of what will follow (we assume that the reader is familiar with the concept of a Lie algebroid). We recall the equations of motion for a non-holonomic mechanical system and take them as the starting point for further generalization in the framework of Lie algebroids, eventually leading to the equations describing a 'non-holonomic Lagrangian system on a Lie algebroid'. Let $L \in C^{\infty}(T Q)$ be the Lagrangian of a non-holonomic system. The constraints are assumed to define a subbundle of the tangent bundle: $\iota: D \rightarrow T Q$, i.e. locally we have that $\iota_{A}^{k}(x) w^{A}=v^{k}$ where $v^{k}$ denote the components of $\mathrm{i}(w)$ wrt the standard basis $\left\{\partial / \partial x^{k}\right\}$ and where $w^{A}$ are the components of $w$ wrt some basis of $D$. A basis of the annihilator space $D^{0}$ is denoted by $\omega^{b}=\omega_{i}^{b} \mathrm{~d} x^{i}$, i.e. $\omega_{i}^{b} \iota_{A}^{i}=0, \forall b, A$. The Lagrange-d'Alembert principle states that the equations of motion for such a non-holonomic system are determined by $\delta \int_{a}^{b} L\left(x^{i}(t), \dot{x}^{i}(t)\right) \mathrm{d} t=0$, where the variations $\delta x^{i}$ should satisfy the constraint, i.e. $\delta x \in D_{x(t)}$ for each $t \in[a, b]$, and moreover $\delta x(a)=\delta x(b)=0$. The induced equations are called the Lagrange-d'Alembert equations:

$$
\left\{\begin{array}{l}
\omega_{i}^{b} \dot{x}^{i}=0, \quad \forall b \\
\frac{\mathrm{d}}{\mathrm{d} t}\left(\frac{\partial L}{\partial \dot{x}^{i}}\right)=\frac{\partial L}{\partial x^{i}}+\lambda_{b}(t) \omega_{i}^{b}(t) \in D_{x(t)}^{0},
\end{array}\right.
$$

for some functions $\lambda_{b}(t)$. It is not hard to see that this system is equivalent to the system:

$$
\left\{\begin{array}{l}
\dot{x}^{i}=\iota_{A}^{i}(x(t)) w^{A}(t), \\
\iota_{A}^{i} \frac{\mathrm{d}}{\mathrm{d} t}\left(\frac{\partial L}{\partial \dot{x}^{i}}\right)=\iota_{A}^{\mathrm{i}} \frac{\partial L}{\partial x^{i}}, \quad \forall A .
\end{array}\right.
$$

We now repeat the above construction for systems on a Lie algebroid. Assume that a local coordinate chart $(U,(x, \mathrm{v}))$ of a Lie algebroid $\tau: \mathrm{V} \rightarrow M$ is given. Given a Lagrangian $L$ on $\mathrm{V}$, the Lagrangian equations on the Lie algebroid are given by

$$
\left\{\begin{array}{l}
\dot{x}^{i}=\rho_{a}^{i}(x) \mathrm{v}^{a}, \\
\frac{\mathrm{d}}{\mathrm{d} t}\left(\frac{\partial L}{\partial \mathrm{v}^{a}}\right)=\rho_{a}^{i} \frac{\partial L}{\partial x^{i}}-C_{a b}^{c} \mathrm{v}^{b} \frac{\partial L}{\partial \mathrm{v}^{c}},
\end{array}\right.
$$

where $\rho_{a}^{i}$ and $C_{a b}^{c}$ are the structure functions of the Lie algebroid. We now assume that W is a subbundle of $\mathrm{V}$ with injection $i: \mathrm{W} \rightarrow \mathrm{V}$. We will use the observation of the previous paragraph: by contracting the unconstrained Lagrangian equations with the components of the injection $\iota_{A}^{a}$ of the constraint distribution into the tangent bundle, the non-holonomic equations can be produced. Assuming that Lagrangian systems constrained to the subbundle $W$ of a 
Lie algebroid $\mathrm{V}$ have a similar behaviour, we can now postulate that the constrained Lagrangian equations are

$$
\left\{\begin{array}{l}
\dot{x}^{i}(t)=\rho_{a}^{i}(x(t)) \mathrm{v}^{a}(t), \\
\mathrm{v}^{a}(t)=i_{A}^{a}(x(t)) w^{A}(t), \\
i_{A}^{a}\left(\frac{\mathrm{d}}{\mathrm{d} t}\left(\frac{\partial L}{\partial \mathrm{v}^{a}}\right)\right)=i_{A}^{a}\left(\rho_{a}^{i} \frac{\partial L}{\partial x^{i}}-C_{a b}^{c} \mathrm{v}^{b} \frac{\partial L}{\partial \mathrm{v}^{c}}\right) .
\end{array}\right.
$$

We can now write the above equations in terms of derivatives of the constrained Lagrangian, defined by $L_{c}\left(x^{i}, w^{A}\right)=L\left(x^{i}, \mathrm{v}^{a}=i_{A}^{a} w^{A}\right)$. Then, along the solution $\mathrm{v}=i \circ w$ of $(4)$, a straightforward calculation leads to:

$$
\begin{aligned}
\frac{\mathrm{d}}{\mathrm{d} t}\left(\frac{\partial L_{c}}{\partial w^{A}}\right) & =\frac{\mathrm{d}}{\mathrm{d} t}\left(i_{A}^{a}\right) \frac{\partial L}{\partial \mathrm{v}^{a}}+i_{A}^{a} \frac{\mathrm{d}}{\mathrm{d} t}\left(\frac{\partial L}{\partial \mathrm{v}^{a}}\right) \\
& =\frac{\partial i_{A}^{a}}{\partial x^{i}} \dot{x}^{i} \frac{\partial L}{\partial \mathrm{v}^{a}}+i_{A}^{a}\left(\rho_{a}^{i} \frac{\partial L}{\partial x^{i}}-C_{a b}^{c} \mathrm{v}^{b} \frac{\partial L}{\partial \mathrm{v}^{c}}\right) .
\end{aligned}
$$

If we define $\lambda_{A}^{i}=\rho_{a}^{i} i_{A}^{a}$ then, since $\partial L_{c} / \partial x^{i}=\partial L / \partial x^{i}+\left(\partial L / \partial v^{c}\right)\left(\partial i_{B}^{c} / \partial x^{i}\right) w^{B}$, it is clear that the curve $(x(t), w(t))$ is a solution to the system:

$$
\left\{\begin{array}{l}
\dot{x}^{i}=\lambda_{A}^{i}(x) w^{A}, \\
\frac{\mathrm{d}}{\mathrm{d} t}\left(\frac{\partial L_{c}}{\partial w^{A}}\right)=\lambda_{A}^{i} \frac{\partial L_{c}}{\partial x^{i}}+w^{B}\left(C_{b a}^{c} i_{B}^{b} i_{A}^{a}-\lambda_{A}^{i} \frac{\partial i_{B}^{c}}{\partial x^{i}}+\lambda_{B}^{i} \frac{\partial i_{A}^{c}}{\partial x^{i}}\right) \frac{\partial L}{\partial \mathrm{v}^{c}} .
\end{array}\right.
$$

In the first part of this paper, we develop all geometric structures required to provide an intrinsic formulation for this system of equations (5). In [14], Martínez presented a solid geometrical framework for Weinstein's systems on Lie algebroids. His approach is very similar to the usual formalism for Euler-Lagrange equations. The important difference is, however, that no longer a vector field is the main geometrical object, but rather a section of a 'prolongation bundle' inducing a unique vector field on the Lie algebroid. In this paper we extend the framework of Martínez to the above constrained systems (5). For that purpose, we first define in section 2 an exterior derivative on a subbundle of a Lie algebroid. Next, we develop the concept of prolongation bundles in section 3, eventually leading to all necessary tools for an intrinsic description of (5) in section 4. In our formalism, the system (5) will be regarded as a section of an appropriate prolongation bundle. With this section a vector field is associated, whose integral curves are precisely the solution of (5). The second part of the paper is devoted to examples from known dynamical systems that allow a formulation in terms of the above equations (cf section 5). These examples only deal with autonomous systems with linear constraints. Finally, we will discuss some of the advantages of our approach and we will indicate some directions for future work.

\section{Exterior derivatives}

A Lie algebroid is a vector bundle $\tau: \mathrm{V} \rightarrow M$ with a real Lie algebra bracket on its set of sections $[\cdot, \cdot]: \operatorname{Sec}(\tau) \times \operatorname{Sec}(\tau) \rightarrow \operatorname{Sec}(\tau)$. Moreover there is a linear bundle map, the so-called anchor map, $\rho: \mathrm{V} \rightarrow T M$ (and its natural extension $\rho: \operatorname{Sec}(\tau) \rightarrow \mathcal{X}(M)$ ) which is related to the bracket in such a way that, for all $\mathrm{s}, \mathrm{r} \in \operatorname{Sec}(\tau), f \in C^{\infty}(M)$

$$
[\mathrm{s}, f \mathrm{r}]=f[\mathrm{~s}, \mathrm{r}]+\rho(\mathrm{s})(f) \mathrm{r}
$$

is satisfied. 
Choose a basis $\left\{\mathrm{e}_{a}\right\}$ for $\operatorname{Sec}(\tau)$ and denote the corresponding local coordinates on $\mathrm{V}$ by $\left(x^{i}, \mathrm{v}^{a}\right)$. Then, the structure functions of the Lie algebroid are smooth functions $\rho_{a}^{i}$ and $C_{a b}^{c}$ on $M$ which satisfy $\rho\left(\mathrm{e}_{a}\right)=\rho_{a}^{i} \frac{\partial}{\partial x^{i}}$ and $\left[\mathrm{e}_{a}, \mathrm{e}_{b}\right]=C_{a b}^{c} \mathrm{e}_{c}$. In Lie algebroid theory, the role of differential forms is played by sections of exterior powers of the dual vector bundle, i.e. skewsymmetric, $C^{\infty}(M)$-linear maps $\omega: \operatorname{Sec}(\tau) \times \cdots \times \operatorname{Sec}(\tau) \rightarrow C^{\infty}(M)$ (with $k$ arguments) will be called $k$-forms on $\operatorname{Sec}(\tau)$ and the set of all such forms will be denoted by $\bigwedge^{k}(\tau)$. The defining properties of a Lie algebroid structure lead to the definition of an exterior derivative on $\bigwedge(\tau)$. Let $\omega \in \bigwedge^{k}(\tau)$, then the $(k+1)$-form $\mathrm{d} \omega$ is given by

$$
\begin{aligned}
\mathrm{d} \omega\left(\mathrm{s}_{1}, \ldots, \mathrm{s}_{k+1}\right) & =\sum_{i=1}^{k+1}(-1)^{i-1} \rho\left(\mathrm{s}_{i}\right)\left(\omega\left(\mathrm{s}_{1}, \ldots, \hat{\mathrm{s}}_{i}, \ldots, \mathrm{s}_{k+1}\right)\right) \\
+ & \sum_{1 \leqslant i<j \leqslant k+1}(-1)^{i+j} \omega\left(\left[\mathrm{s}_{i}, \mathrm{~s}_{j}\right], \mathrm{s}_{1}, \ldots, \hat{\mathrm{s}}_{i}, \ldots, \hat{\mathrm{s}}_{j}, \ldots, \mathrm{s}_{k+1}\right) .
\end{aligned}
$$

The operator $\mathrm{d}$ has the property $\mathrm{d}\left(\omega_{1} \wedge \omega_{2}\right)=\mathrm{d} \omega_{1} \wedge \omega_{2}+(-1)^{k_{1}} \omega_{1} \wedge \mathrm{d} \omega_{2}$, with $k_{1}$ the degree of $\omega_{1}$, and moreover $d^{2}=0$. Locally, $d x^{i}=\rho_{a}^{i} \mathrm{e}^{a}$ and $d \mathrm{e}^{c}=-\frac{1}{2} C_{a b}^{c} \mathrm{e}^{a} \wedge \mathrm{e}^{b}$, where the set $\left\{\mathrm{e}^{a}\right\}$ stands for the basis of $\operatorname{Sec}\left(\tau^{*}\right)$ which is dual to $\left\{\mathrm{e}_{a}\right\}$. More details on the properties of the exterior derivative can be found in $[9,13,14]$.

Suppose now that a vector subbundle $\mu: \mathrm{W} \rightarrow M$ of $\tau$ is given. It is obvious that each coordinate system $\left(x^{i}, w^{A}\right)$ on $\mathrm{W}$ can be extended to a coordinate system $\left(x^{i}, \mathrm{v}^{A}=w^{A}, \mathrm{v}^{\alpha}\right)$ on V. However, in what follows, it will be more convenient to consider the basis $\left\{\mathrm{e}_{a}\right\}$ of $\operatorname{Sec}(\tau)$ to be a priori given. Then, the sections of an arbitrary basis $\left\{e_{A}\right\}$ of $\operatorname{Sec}(\mu)$, can be written as $e_{A}=i_{A}^{a} \mathrm{e}_{a}$ and the injection can be denoted by $i: \mathrm{W} \rightarrow \mathrm{V} ;\left(x^{i}, w^{A}\right) \mapsto\left(x^{i}, \mathrm{v}^{a}=i_{A}^{a} w^{A}\right)$. We will use $\lambda$ for the restriction of $\rho$ to $\mathrm{W}: \lambda=\rho \circ i: \mathrm{W} \rightarrow T M ;\left(x^{i}, w^{A}\right) \mapsto\left(x^{i}, \dot{x}^{i}=\right.$ $\left.\lambda_{A}^{i} w^{A}=\rho_{a}^{i} i_{A}^{a} w^{A}\right)$.

Forms on $\operatorname{Sec}(\tau)$ can be pulled back to forms on $\operatorname{Sec}(\mu)$. Indeed, if $\omega$ is a $k$-form then $i^{*} \omega$, defined by

$$
i^{*} \omega\left(W_{1}, \ldots, W_{k}\right)=\omega\left(i W_{1}, \ldots, i W_{k}\right), \quad W_{i} \in \operatorname{Sec}(\mu)
$$

is a $k$-form on $\operatorname{Sec}(\mu)$. By composing the exterior derivative $\mathrm{d}$ with $i^{*}$ we can define a mapping from $\bigwedge^{k}(\tau)$ to $\bigwedge^{k+1}(\mu)$, which is denoted by $\delta$. Thus, if $\omega$ is a $k$-form on $\operatorname{Sec}(\tau)$, then

$$
\delta \omega=i^{*} \mathrm{~d} \omega,
$$

is a $(k+1)$-form on $\operatorname{Sec}(\mu)$. The operator $\delta$ satisfies the rule

$$
\delta\left(\omega_{1} \wedge \omega_{2}\right)=\delta \omega_{1} \wedge i^{*} \omega_{2}+(-1)^{k_{1}} i^{*} \omega_{1} \wedge \delta \omega_{2},
$$

and could be called a derivative along $i$ for this reason, but we will simply refer to $\delta$ as an exterior derivative. Obviously, $\delta \circ \mathrm{d}=0$. In the above introduced coordinates,

$$
\delta x^{i}=\rho_{a}^{i} i_{A}^{a} e^{A}=\lambda_{A}^{i} e^{A} \quad \text { and } \quad \delta \mathrm{e}^{a}=-\frac{1}{2} D_{B C}^{a} e^{B} \wedge e^{C},
$$

where $D_{B C}^{a}=C_{b c}^{a} i_{B}^{b} i_{C}^{c}$.

To illustrate the above notions, we look at the case where $\mathrm{W}$ is a Lie subalgebroid of $\mathrm{V}$. A more general definition of a Lie subalgebroid $\mathrm{W} \rightarrow N$ of $\mathrm{V} \rightarrow M$ can be found in [10]. Here, we will only consider the case that the base manifolds coincide. A Lie algebroid $\mu: \mathrm{W} \rightarrow M$ (with anchor map $\lambda$ and structure functions $D_{A B}^{C}$ ) is a Lie subalgebroid of $\tau: \mathrm{V} \rightarrow M$ (with anchor map $\rho$ ) if there exists a injective morphism $i: \mathrm{W} \rightarrow \mathrm{V}$ such that $\lambda=\rho \circ i$ and

$$
i\left[W_{1}, W_{2}\right]=\left[i\left(W_{1}\right), i\left(W_{2}\right)\right], \quad W_{i} \in \operatorname{Sec}(\mu) .
$$

In coordinates the above expression reads

$$
i_{C}^{c} D_{A B}^{C}=D_{A B}^{c}+\lambda_{A}^{i} \frac{\partial i_{B}^{c}}{\partial x^{i}}-\lambda_{B}^{i} \frac{\partial i_{A}^{c}}{\partial x^{i}} .
$$


There are now three exterior derivatives around: $\mathrm{d}, \delta$ and the exterior derivative $\mathrm{d}^{\mu}$ on the Lie algebroid $\mu$. The condition (8) can equivalently be written as

$$
\mathrm{d}^{\mu} \circ i^{*}=i^{*} \circ \mathrm{d}=\delta .
$$

Indeed, for functions on $M$ it is obviously satisfied, while for 1-forms $\theta \in \bigwedge^{1}(\tau)$

$$
\mathrm{d}^{\mu} i^{*} \theta\left(W_{1}, W_{2}\right)=\lambda\left(W_{1}\right) \theta\left(i W_{2}\right)-\lambda\left(W_{2}\right) \theta\left(i W_{1}\right)-\theta\left(i\left[W_{1}, W_{2}\right]\right) .
$$

This is $\mathrm{d} \theta\left(i W_{1}, i W_{2}\right)$ if and only if (8) is satisfied. The proof then follows from induction. It is now also obvious that for Lie subalgebroids

$$
\mathrm{d}^{\mu} \circ \delta=i^{*} \circ \mathrm{d}^{2}=\left(\mathrm{d}^{\mu}\right)^{2} \circ i^{*}=0 .
$$

\section{Prolongation bundles}

As stated in the introduction, an important role in our description will be played by the so-called prolongation bundle associated with a Lie algebroid. Sections of such bundles will eventually lead to the vector field on the Lie algebroid that generates the equations in (5). Furthermore, these bundles allow a generalization of all intrinsic objects defined on the tangent bundle needed to write down the standard Lagrange equations. We start with defining the prolongation of an arbitrary bundle, and then continue with the introduction of all the structures that lead to an intrinsic formulation of (5).

Let $\pi: E \rightarrow M$ be an arbitrary fibre bundle. The prolongation of $\pi$ by an anchored vector bundle $\tau: \mathrm{V} \rightarrow M$ is the (vector) bundle $\pi^{\rho}: T^{\rho} E \rightarrow E$. Here, the total space $T^{\rho} E$ is the pullback manifold $\rho^{*} T E=\left\{\left(\mathrm{v}, X_{e}\right) \in \mathrm{V} \times T E \mid \rho(\mathrm{v})=T \pi\left(X_{e}\right)\right\}$. The natural projections of $T^{\rho} E$ onto $\mathrm{V}$ and $T E$ will be denoted by $\pi^{2}$ and $\rho^{\pi}$, respectively (see the diagram below). On the other hand, the bundle projection $\pi^{\rho}$ is given by $\tau_{E} \circ \rho^{1}$, i.e. $\pi^{\rho}\left(\mathrm{v}, X_{e}\right)=e$ (see also e.g. $[9,10,14,16])$.

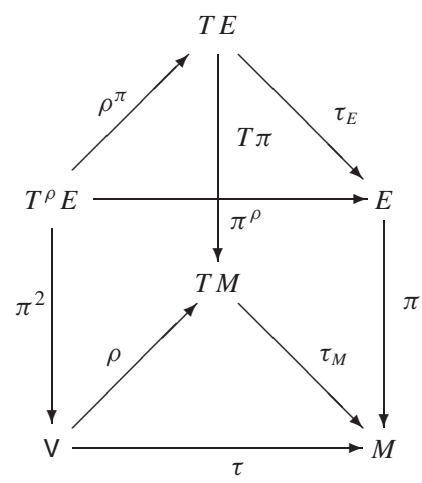

Suppose we fix a bundle adapted coordinate chart $\left(U,\left(x^{i}, y^{\alpha}\right)\right)$ about a point $e \in E$. It is not difficult to see that $\left\{\mathcal{X}_{a}, \mathcal{Y}_{\alpha}\right\}$, with

$\mathcal{X}_{a}(e)=\left(\mathrm{e}_{a}(\pi(e)),\left.\rho_{a}^{i}(x) \frac{\partial}{\partial x^{i}}\right|_{e}\right) \quad$ and $\quad \mathcal{Y}_{\alpha}(e)=\left(0(\pi(e)),\left.\frac{\partial}{\partial y^{\alpha}}\right|_{e}\right)$,

is a basis of $\operatorname{Sec}\left(\pi^{\rho}\right)$, induced by the basis $\left\{\mathrm{e}_{a}\right\}$ of $\operatorname{Sec}(\tau)$ and the basis $\left\{\frac{\partial}{\partial x^{i}}, \frac{\partial}{\partial y^{\alpha}}\right\}$ of $\mathcal{X}(E)$. The set $\mathcal{V}^{\rho} E=\left\{\left(0, X_{e}\right) \in T^{\rho} E\right\}$ is called the bundle of vertical elements of $T^{\rho} E$. A basis for the set of vertical sections $\operatorname{Ver}\left(\pi^{\rho}\right)$ is given by the $\left\{\mathcal{Y}_{\alpha}\right\}$. 
Suppose now that $\tau: \mathrm{V} \rightarrow M$ is a Lie algebroid. The Lie algebroid structure on $\tau$ can be naturally extended to a Lie algebroid structure on the prolongation bundle $\pi^{\rho}: T^{\rho} E \rightarrow E$. The anchor map of this Lie algebroid is $\rho^{\pi}: T^{\rho} E \rightarrow T E$, with

$$
\rho^{\pi}\left(\mathcal{X}_{a}\right)=\rho_{a}^{i} \frac{\partial}{\partial x^{i}} \quad \text { and } \quad \rho^{\pi}\left(\mathcal{Y}_{\alpha}\right)=\frac{\partial}{\partial y^{\alpha}}
$$

and the bracket is given by

$$
\left[\mathcal{X}_{a}, \mathcal{X}_{b}\right]=C_{a b}^{c} \mathcal{X}_{c}, \quad\left[\mathcal{X}_{a}, \mathcal{Y}_{\beta}\right]=0, \quad\left[\mathcal{Y}_{\alpha}, \mathcal{Y}_{\beta}\right]=0
$$

(for more details, see e.g. [9, 14]).

Let $\mu: \mathrm{W} \rightarrow M$ be a subbundle of $\tau$. We will need three different prolongation bundles. The first is the one where $\pi$ is also $\tau: \mathrm{V} \rightarrow M$, that is $\tau^{\rho}: T^{\rho} \mathrm{V} \rightarrow \mathrm{V}$. The basis (11) will, in this case, be denoted by $\left\{\mathcal{X}_{a}, \mathcal{V}_{a}\right\}$ and we will use $\left\{\mathcal{X}^{a}, \mathcal{V}^{a}\right\}$ for the basis of $\operatorname{Sec}\left(\left(\tau^{\rho}\right)^{*}\right)$ that is dual to $\left\{\mathcal{X}_{a}, \mathcal{V}_{a}\right\}$. Since $\tau^{\rho}$ has a Lie algebroid structure, it also has an exterior derivative d, locally characterized by the relations

$\mathrm{d} x^{i}=\rho_{a}^{i} \mathcal{X}^{a}, \quad \mathrm{dv}^{a}=\mathcal{V}^{a}, \quad \mathrm{~d} \mathcal{X}^{c}=-\frac{1}{2} C_{a b}^{c} \mathcal{X}^{a} \wedge \mathcal{X}^{b} \quad$ and $\quad \mathrm{d} \mathcal{V}^{a}=0$.
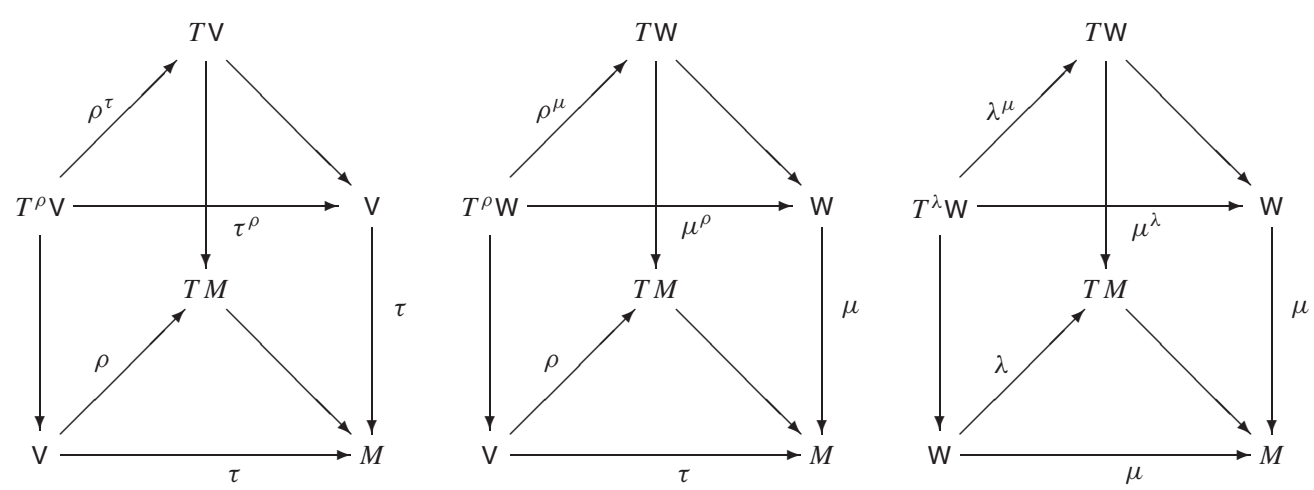

The second prolongation of interest is $\mu^{\rho}: T^{\rho} \mathrm{W} \rightarrow \mathrm{W}$. Again, $\mu^{\rho}$ is a Lie algebroid with a corresponding exterior derivative, denoted by $\tilde{\mathrm{d}}$. If $\left\{\tilde{\mathcal{X}}_{a}, \tilde{\mathcal{W}}_{A}\right\}$ stands for the basis (11) in this situation, then

$\tilde{\mathrm{d}} x^{i}=\rho_{a}^{i} \tilde{\mathcal{X}}^{a}, \quad \tilde{\mathrm{d}} w^{A}=\tilde{\mathcal{W}}^{A}, \quad \tilde{\mathrm{d}} \tilde{\mathcal{X}}^{c}=-\frac{1}{2} C_{a b}^{c} \tilde{\mathcal{X}}^{a} \wedge \tilde{\mathcal{X}}^{b} \quad$ and $\quad \tilde{\mathrm{d}} \tilde{\mathcal{V}}^{A}=0$,

$\mu^{\rho}$ is in fact a Lie subalgebroid of $\tau^{\rho}$ (in the more general sense of [10], see also [9]).

Last but not the least, we will also need the prolongation $\mu^{\lambda}: T^{\lambda} \mathrm{W} \rightarrow \mathrm{W}$, which is a vector subbundle of the Lie algebroid $T^{\rho} \mathrm{W} \rightarrow \mathrm{W}$. Let $\left\{\mathcal{X}_{A}, \mathcal{W}_{A}\right\}$ stand for the basis of $\operatorname{Sec}\left(\mu^{\lambda}\right)$, induced by the bases $\left\{e_{A}\right\}$ and $\left\{\frac{\partial}{\partial x^{i}}, \frac{\partial}{\partial w^{A}}\right\}$. Then, the injection is given by the map $I: T^{\lambda} \mathrm{W} \rightarrow T^{\rho} \mathrm{W} ;\left(w_{1}, X_{w_{2}}\right) \mapsto\left(i\left(w_{1}\right), X_{w_{2}}\right)$ which, locally, is of the form

$$
I\left(\mathcal{X}_{A}\right)=i_{A}^{a} \tilde{\mathcal{X}}_{a} \quad \text { and } \quad I\left(\mathcal{W}_{A}\right)=\tilde{\mathcal{W}}_{A} .
$$

In view of the discussion of the previous section, we can use $I$ to introduce an 'exterior derivative' $\delta$, defined as in (7), which will map forms on $\operatorname{Sec}\left(\mu^{\rho}\right)$ to forms on $\operatorname{Sec}\left(\mu^{\lambda}\right)$. Locally,

$\delta x^{i}=\lambda_{A}^{i} \mathcal{X}^{A}, \quad \delta w^{A}=\mathcal{W}^{A}, \quad \delta \tilde{\mathcal{X}}^{c}=-\frac{1}{2} D_{A B}^{c} \mathcal{X}^{A} \wedge \mathcal{X}^{B} \quad$ and $\quad \delta \tilde{\mathcal{W}}^{A}=0$. 
A second important map $T^{\rho} i: T^{\rho} \mathrm{W} \rightarrow T^{\rho} \mathrm{V}$ is the linear bundle map over $i: \mathrm{W} \rightarrow \mathrm{V}$, given by $T^{\rho} i\left(\mathrm{v}, X_{w}\right)=\left(\mathrm{v}, T_{w} i\left(X_{w}\right)\right)$. Let an element $Z \in T_{w}^{\rho} \mathrm{W}$ be given by $Z=$ $X^{a} \tilde{\mathcal{X}}_{a}(w)+W^{A} \tilde{\mathcal{W}}_{A}(w)$, then

$$
T^{\rho} i(Z)=X^{a} \mathcal{X}_{a}(i(w))+\left(\rho_{b}^{i} X^{b} \frac{\partial i_{A}^{a}}{\partial x^{i}} w^{A}+i_{A}^{a} W^{A}\right) \mathcal{V}_{a}(i(w))
$$

Both prolongations $\tau^{\rho}: T^{\rho} \mathrm{V} \rightarrow \mathrm{V}$ and $\mu^{\lambda}: T^{\lambda} \mathrm{W} \rightarrow \mathrm{W}$ carry further interesting canonical objects. For example, there exists a naturally defined vertical lift ${ }^{V}: \tau^{*} \mathrm{~V} \rightarrow \mathcal{V}^{\rho} \mathrm{V} \subset T^{\rho} \mathrm{V}$. Indeed, for $(\mathrm{a}, \mathrm{v}) \in \tau^{*} \mathrm{~V}$, we can define first a vertical element $\mathrm{v}_{\mathrm{a}}^{v} \in T_{\mathrm{a}} \mathrm{V}$ by means of its action on functions $f \in C^{\infty}(\mathrm{V})$,

$$
\mathrm{v}_{\mathrm{a}}^{v}(f)=\left.\frac{\mathrm{d}}{\mathrm{d} t} f(\mathrm{a}+t \mathrm{v})\right|_{t=0} .
$$

Then, the element $\mathrm{v}_{\mathrm{a}}^{V} \in \mathcal{V}^{\rho} \mathrm{V}$ is defined as $\left(0, \mathrm{v}_{\mathrm{a}}^{v}\right)$. We have $\mathrm{e}_{a}^{V}=\mathcal{V}_{a}$. Evidently, there exists a similar notion for the prolongation $\mu^{\lambda}$, here the vertical lift is a map ${ }^{v}: \mu^{*} \mathrm{~W} \rightarrow \mathcal{V}^{\lambda} \mathrm{W} \subset T^{\lambda} \mathrm{W}$. Next, there are also two so-called vertical endomorphisms $S^{\tau}={ }^{v} \circ j_{1}: \operatorname{Sec}\left(\tau^{\rho}\right) \rightarrow \operatorname{Ver}\left(\tau^{\rho}\right)$, and $S^{\mu}={ }^{v} \circ j_{2}: \operatorname{Sec}\left(\mu^{\lambda}\right) \rightarrow \operatorname{Ver}\left(\mu^{\lambda}\right)$, where $j_{1}$ stands for the projection $\left(\mathrm{v}_{2}, X_{\mathrm{v}_{1}}\right) \in T^{\rho} \mathrm{V} \mapsto\left(\mathrm{v}_{1}, \mathrm{v}_{2}\right)$, and $j_{2}: T^{\lambda} \mathrm{W} \rightarrow \mu^{*} \mathrm{~W}$ can be defined analogously. In the above-introduced bases, $S^{\tau}=\mathcal{X}^{a} \otimes \mathcal{V}_{a}$ and $S^{\mu}=\mathcal{X}^{A} \otimes \mathcal{W}_{A}$. Remark that, although the projection $j_{3}: T^{\rho} \mathrm{W} \rightarrow \mu^{*} \mathrm{~V}$ can easily be defined, there exists no vertical endomorphism on $T^{\rho} \mathrm{W}$ because there is no vertical lift which maps elements in $\mu^{*} \mathrm{~V}$ to $\mathcal{V}^{\rho} \mathrm{W}$. Two final important objects are the Liouville sections $\mathcal{C}^{\tau}=\mathrm{v}^{a} \mathcal{V}_{a} \in \operatorname{Sec}\left(\tau^{\rho}\right)$ and $\mathcal{C}^{\mu}=w^{A} \mathcal{W}_{A} \in \operatorname{Sec}\left(\mu^{\lambda}\right)$.

\section{Lagrange equations on a subbundle of a Lie algebroid}

In this section we formulate the equations for a non-holonomic system on a Lie algebroid. The main purpose is to arrive at an equation that uniquely determines a vector field on $\mathrm{W}$ whose integral curves are precisely the solutions to the constrained equations (5). We will only deal with regular Lagrangians $L \in C^{\infty}(\mathrm{V})$, i.e. we will assume that the matrix $\left(\partial^{2} L / \partial \mathrm{v}^{\alpha} \partial \mathrm{v}^{\beta}\right)$ is regular at every point.

Let us recall first briefly Martínez's description of Lagrangian systems (3) on a Lie algebroid. An important subclass of sections of the prolongation bundle $\tau^{\rho}: T^{\rho} \mathrm{V} \rightarrow \mathrm{V}$ are the so-called pseudo-SodEs [16, 17], or simply SodEs in $[9,14]$. They are sections $\Gamma$ of $\tau^{\rho}$ such that $\tau^{2} \circ \Gamma=i d\left(\tau^{2}\right.$ is the projection $\left.(\mathrm{v}, V) \in T^{\rho} \mathrm{V} \mapsto \mathrm{v} \in \mathrm{V}\right)$. Locally, a pseudo-SodE is of the form

$$
\Gamma=\mathrm{v}^{a} \mathcal{X}_{a}+f^{a} \mathcal{V}_{a}
$$

It is not difficult to see that pseudo-SoDEs are in a one-to-one correspondence with vector fields $X$ on $\mathrm{V}$ with the property that $\rho(\mathrm{v})=T \tau(X(\mathrm{v}))$. Keeping this in mind, by defining a pseudo-SodE, one can give an intrinsic description of the equations (3). A regular Lagrangian $L$ defines a function

$$
E_{L}=\rho^{\tau}\left(C^{\tau}\right) L-L=\mathrm{v}^{a} \frac{\partial L}{\partial \mathrm{v}^{a}}(\mathrm{v})-L(\mathrm{v})
$$

on $\mathrm{V}$ and a 1 -form

$$
\theta_{L}=S^{\tau}(\mathrm{d} L)=\frac{\partial L}{\partial \mathrm{v}^{a}}(\mathrm{v}) \mathcal{X}^{a}
$$

on $\operatorname{Sec}\left(\tau^{\rho}\right)$. The dynamics are then given by a pseudo-Sode $\Gamma$ of the prolongation $\tau^{\rho}$ that solves the equation

$$
i_{\Gamma} \mathrm{d} \theta_{L}=-\mathrm{d} E_{L} .
$$


Solutions of the equations (3) are then nothing but integral curves of the associated vector field $\rho^{\tau}(\Gamma) \in \mathcal{X}(\mathrm{V})$.

For the constrained systems (5), we wish to preserve, as much as possible, the structure of the equation (14). By analogy, it is clear that we should represent the dynamics by a section of the prolongation bundle $\mu^{\lambda}: T^{\lambda} \mathrm{W} \rightarrow \mathrm{W}$. It is easy to see that pseudo-SODEs $\Gamma$ on this bundle have locally the form

$$
\Gamma=w^{A} \mathcal{X}_{A}+f^{A} \mathcal{W}_{A} .
$$

Crucial in (14) is the exterior derivative $\mathrm{d}$ of the prolongation $\tau^{\rho}$. Unfortunately, the prolongation bundle $\mu^{\lambda}$ does not carry a Lie algebroid structure and therefore there is no available exterior derivative. The next best thing is the above-introduced operator $\delta$. As a consequence, the analogues $\tilde{E}_{L}$ and $\tilde{\theta}_{L}$ of the function $E_{L}$ and the 1-form $\theta_{L}$ to the constrained case should be sought, respectively, among the functions on $\mathrm{W}$ and the 1-forms on $\operatorname{Sec}\left(\mu^{\rho}\right)$. For $\tilde{E}_{L}$ we can simply take the restriction of $E_{L}$ to W. If we define the constrained Lagrangian $L_{c} \in C^{\infty}(\mathrm{W})$ as the restriction of $L$ to $\mathrm{W}, L_{c}(w)=L(i(w))$, then it is easy to see that $\tilde{E}_{L}$ can also be given by

$$
\tilde{E}_{L}=\lambda^{\mu}\left(C^{\mu}\right)\left(L_{c}\right)-L_{c}=w^{A} \frac{\partial L_{c}}{\partial w^{A}}(w)-L_{c}(w) \in C^{\infty}(\mathrm{W})
$$

The construction of $\theta_{L}$ in (13) can, however, not directly be translated to $\mu^{\rho}$. Indeed, although $\tilde{\mathrm{d}} L_{c}$ is a well-defined 1-form on $\operatorname{Sec}\left(\mu^{\rho}\right)$, there exists no vertical endomorphism on $\operatorname{Sec}\left(\mu^{\rho}\right)$. On the other hand, we can also start from $\delta L_{c}$, which is a 1-form on $\operatorname{Sec}\left(\mu^{\lambda}\right)$, but its image under the vertical endomorphism $S^{\mu}$ of $\mu^{\lambda}$ gives us a 1 -form

$$
\theta_{L_{c}}=S^{\mu}\left(\delta L_{c}\right)=\frac{\partial L_{c}}{\partial w^{A}}(w) \mathcal{X}^{A}=i_{A}^{a} \frac{\partial L}{\partial \mathrm{v}^{a}}(i(w)) \mathcal{X}^{A}
$$

on $\operatorname{Sec}\left(\mu^{\lambda}\right)$ and not on $\operatorname{Sec}\left(\mu^{\rho}\right)$, as required. Having found no direct construction on $\mu^{\rho}$, it seems appropriate to take one more step backwards (wrt the diagrams of the previous section). We will use a suitable restriction of $\theta_{L}$ to $\mu^{\rho}$.

Definition 1. The Poincaré-Cartan 1-form $\tilde{\theta}_{L}$ is defined as the 1 -form $\left(T^{\rho} i\right)^{*}\left(\theta_{L}\right)$ on $\operatorname{Sec}\left(\mu^{\rho}\right)$, i.e.

$$
\tilde{\theta}_{L}(w)(\mathrm{v}, W)=\theta_{L}(i(w))\left(T^{\rho} i(\mathrm{v}, W)\right),
$$

or, locally, $\tilde{\theta}_{L}=\frac{\partial L}{\partial \mathrm{v}^{a}}(i(w)) \tilde{\mathcal{X}}^{a}$.

The energy function $\tilde{E}_{L}$ is the restriction of $E_{L}$ to $\mathrm{W}$.

From the coordinate expressions it is easy to see that $\theta_{L_{c}}=I^{*}\left(\tilde{\theta}_{L}\right)$, i.e.

$$
\theta_{L_{c}}\left(w_{1}\right)\left(w_{2}, W\right)=\tilde{\theta}_{L}\left(w_{1}\right)\left(I\left(w_{2}, W\right)\right) .
$$

We now have all the ingredients for a coordinate free description of (5).

Definition 2. A Lagrangian system on $\mu^{\lambda}: T^{\lambda} \mathrm{W} \rightarrow \mathrm{W}$ is a pseudo-SodE $\Gamma \in \operatorname{Sec}\left(\mu^{\lambda}\right)$ that solves the equation

$$
i_{\Gamma} \delta \tilde{\theta}_{L}=-\delta \tilde{E}_{L}
$$

It can easily be checked that (15) gives indeed the correct equations (5). First, one can calculate that

$\delta \tilde{\theta}_{L}=\left(\lambda_{A}^{i} i_{B}^{b} \frac{\partial^{2} L}{\partial x^{i} \partial \mathbf{v}^{b}}+\lambda_{A}^{i} i_{B}^{b} \frac{\partial i_{C}^{c}}{\partial x^{i}} w^{C} \frac{\partial^{2} L}{\partial \mathbf{v}^{b} \partial \mathbf{v}^{c}}-\frac{1}{2} D_{A B}^{c} \frac{\partial L}{\partial \mathbf{v}^{c}}\right) \mathcal{X}^{A} \wedge \mathcal{X}^{B}-i_{B}^{b} i_{A}^{a} \frac{\partial^{2} L}{\partial \mathbf{v}^{a} \partial \mathbf{v}^{b}} \mathcal{X}^{A} \wedge \mathcal{W}^{B}$ 
and

$\delta \tilde{E}_{L}=\left(w^{C} i_{C}^{a} \frac{\partial L^{2}}{\partial x^{i} \partial \mathrm{v}^{a}}+w^{B} w^{C} i_{C}^{b} \frac{\partial i_{B}^{a}}{\partial x^{i}} \frac{\partial L^{2}}{\partial \mathrm{v}^{a} \partial \mathrm{v}^{b}}-\frac{\partial L}{\partial x^{i}}\right) \lambda_{A}^{i} \mathcal{X}^{A}+w^{A} i_{A}^{a} i_{B}^{b} \frac{\partial L^{2}}{\partial \mathrm{v}^{a} \partial \mathrm{v}^{b}} \mathcal{W}^{B}$.

The coefficients $f^{A}$ of $\Gamma$ should therefore satisfy

$\frac{\partial^{2} L}{\partial \mathrm{v}^{a} \partial x^{i}} i_{B}^{a} \lambda_{A}^{i} w^{A}+\frac{\partial^{2} L}{\partial \mathrm{v}^{a} \partial \mathbf{v}^{b}}\left(w^{A} w^{C} i_{B}^{a} \lambda_{C}^{i} \frac{\partial i_{A}^{b}}{\partial x^{i}}+i_{B}^{a} i_{A}^{b} f^{A}\right)-\frac{\partial L}{\partial x^{i}} \lambda_{B}^{i}-\frac{\partial L}{\partial \mathbf{v}^{c}} D_{B A}^{c} w^{A}=0$.

The dynamics are given by the equations of the integral curves of $X=\lambda^{\mu}(\Gamma)=w^{A} \lambda_{A}^{i} \frac{\partial}{\partial x^{i}}+$ $f^{A} \frac{\partial}{\partial w^{A}}$, i.e. they are solutions of

$$
\left\{\begin{array}{l}
\dot{x}^{i}=\lambda_{A}^{i}(x) w^{A}, \\
\dot{w}^{A}=f^{A}(x, w) .
\end{array}\right.
$$

Along such solutions, we find that

$\frac{\mathrm{d}}{\mathrm{d} t}\left(\frac{\partial L_{c}}{\partial w^{A}}\right)=\left(\frac{\partial^{2} L}{\partial \mathrm{v}^{a} \partial x^{i}} i_{A}^{a}+\frac{\partial L}{\partial \mathrm{v}^{a}} \frac{\partial i_{A}^{a}}{\partial x^{i}}\right) \lambda_{B}^{i} w^{B}+\frac{\partial^{2} L}{\partial \mathrm{v}^{a} \partial \mathrm{v}^{b}}\left(w^{B} w^{C} i_{A}^{a} \lambda_{C}^{i} \frac{\partial i_{B}^{b}}{\partial x^{i}}+i_{B}^{b} i_{A}^{a} f^{B}\right)$

and

$$
\frac{\partial L_{c}}{\partial x^{i}}=\frac{\partial L}{\partial x^{i}}+\frac{\partial L}{\partial \mathrm{v}^{a}} \frac{\partial i_{A}^{a}}{\partial x^{i}} w^{A} .
$$

After plugging this information into (16), (17) becomes exactly (5)

$$
\left\{\begin{array}{l}
\dot{x}^{i}=\lambda_{A}^{i}(x) w^{A}, \\
\frac{\mathrm{d}}{\mathrm{d} t}\left(\frac{\partial L_{c}}{\partial w^{A}}\right)=\lambda_{A}^{i} \frac{\partial L_{c}}{\partial x^{i}}+w^{B}\left(D_{B A}^{c}-\lambda_{A}^{i} \frac{\partial i_{B}^{c}}{\partial x^{i}}+\lambda_{B}^{i} \frac{\partial i_{A}^{c}}{\partial x^{i}}\right) \frac{\partial L}{\partial v^{c}} .
\end{array}\right.
$$

\section{Examples}

The main example of this section shows that non-holonomic mechanical systems with symmetry admit a formulation in the above framework after reduction. The first two examples are straightforward.

\subsection{Lagrangian systems on Lie algebroids}

If $\mu: \mathrm{W} \rightarrow M$ is exactly the Lie algebroid $\tau: \mathrm{V} \rightarrow M$, then $i_{b}^{a}=\delta_{b}^{a}$ and $L_{c}=L$. The equations (18) are then exactly (3). Examples of such systems can be found e.g. in rigid body dynamics (see e.g. [14] for a worked-out example about the heavy top), control theory [15] or Lagrangian systems with symmetry on principal fibre bundles [6, 24].

\subsection{Lagrangian equations on Lie subalgebroids}

With the help of (9), it is easy to see that in this case, $\left(D_{B A}^{c}-\lambda_{A}^{i} \frac{\partial i_{B}^{c}}{\partial x^{i}}+\lambda_{B}^{i} \frac{\partial i_{A}^{c}}{\partial x^{i}}\right) \frac{\partial L}{\partial v^{c}}=D_{B A}^{C} \frac{\partial L_{c}}{\partial w^{c}}$. Equations (18) then become exactly equations (3) for the constrained Lagrangian $L_{c}$ on the Lie algebroid $\mu: \mathrm{W} \rightarrow M$. This is also clear from expression (15). Indeed, in this case $\mu^{\lambda}: T^{\lambda} \mathrm{W} \rightarrow \mathrm{W}$ inherits the Lie algebroid structure form $\mu$ and has also an exterior derivative $\mathrm{d}^{\mu}$ which acts on functions and forms in the following way:

$\mathrm{d}^{\mu} x^{i}=\lambda_{A}^{i} \mathcal{X}^{A}, \quad \mathrm{~d}^{\mu} w^{A}=\mathcal{W}^{A}, \quad \mathrm{~d}^{\mu} \mathcal{X}^{C}=-\frac{1}{2} D_{A B}^{C} \mathcal{X}^{A} \wedge \mathcal{X}^{B} \quad$ and $\quad \mathrm{d}^{\mu} \mathcal{W}^{A}=0$. 
Obviously $\delta E_{L_{c}}=\mathrm{d}^{\mu} E_{L_{c}}$. Moreover, due to (9),

$$
\begin{aligned}
\delta \tilde{\theta}_{L} & =\delta\left(\frac{\partial L}{\partial \mathrm{v}^{a}}\right) \wedge I^{*}\left(\tilde{\mathcal{X}}^{a}\right)+I^{*}\left(\frac{\partial L}{\partial \mathrm{v}^{a}}\right) \delta\left(\tilde{\mathcal{X}}^{a}\right) \\
& =\mathrm{d}^{\mu}\left(\frac{\partial L}{\partial \mathrm{v}^{a}}\right) \wedge i_{A}^{a} \mathcal{X}^{A}-\frac{1}{2} \frac{\partial L}{\partial \mathrm{v}^{a}} D_{A B}^{a} \mathcal{X}^{A} \wedge \mathcal{X}^{B} \\
& =\mathrm{d}^{\mu}\left(\frac{\partial L}{\partial \mathrm{v}^{a}}\right) \wedge i_{A}^{a} \mathcal{X}^{A}-\frac{1}{2} \frac{\partial L}{\partial \mathrm{v}^{a}} i_{C}^{a} D_{A B}^{C} \mathcal{X}^{A} \wedge \mathcal{X}^{B} \\
& =\mathrm{d}^{\mu}\left(\frac{\partial L}{\partial \mathrm{v}^{a}}\right) \wedge i_{A}^{a} \mathcal{X}^{A}-\frac{1}{2} \frac{\partial L_{c}}{\partial w^{C}} D_{A B}^{C} \mathcal{X}^{A} \wedge \mathcal{X}^{B}=\mathrm{d}^{\mu} \theta_{L_{c}}
\end{aligned}
$$

Therefore (15) is indeed $i_{\Gamma} d^{\mu} \theta_{L_{c}}=-\mathrm{d}^{\mu} E_{L_{c}}$.

\subsection{Non-holonomic systems}

In the introduction we have already defined the Lagrange-d'Alembert equations describing a non-holonomic mechanical system. We will use similar notations. Suppose $Q$ is the configuration space of a mechanical system that is subject to some kinematic (linear) constraints. If the constraint distribution is $m$-dimensional, then, due to the regularity condition of $D$, we can express $m$ velocities $\dot{s}^{\alpha}$ defined by $\dot{x}^{i}=\left(\dot{r}^{I}, \dot{s}^{\alpha}\right)$, up to a renumbering, in terms of the others

$$
\dot{s}^{\alpha}=-A_{I}^{\alpha}(x) \dot{r}^{I}
$$

Let $\iota: D \rightarrow T Q$, then it is given by $\left(s^{\alpha}, r^{I}, \dot{r}^{I}\right) \mapsto\left(s^{\alpha}, r^{I}, \dot{r}^{I}, \dot{s}^{\alpha}=-A_{I}^{\alpha} \dot{r}^{I}\right)$. Before continuing, we wish to make the following remark: in some geometrical models that treat non-holonomic systems it is sometimes further assumed that there exists a bundle structure of the configuration space $Q$ over some manifold $N$ such that $D$ is the horizontal distribution $H \subset T Q$ of a connection on $Q \rightarrow N$ (this can always be done locally). This additional assumption is not necessary for our purposes. The purpose of this section is to show that the equations for non-holonomic mechanical systems that can be found in e.g. [2], fit into the framework presented above.

We can take $\mathrm{V}$ to be $T Q$, equipped with the natural Lie algebroid structure: the anchor map $\rho$ is the identity and the Lie algebroid bracket is the usual Lie bracket of vector fields. Then $\tau^{\rho}$ is nothing but $T T Q \rightarrow T Q$. The subbundle $\mathrm{W}$ is precisely the distribution $D$, i.e. $i=\iota$. Likewise, $\mu^{\rho}$ becomes simply $T \mathrm{~W} \rightarrow \mathrm{W}$. The Poincaré-Cartan 1 -form $\tilde{\theta}_{L}$ is, in this case, the pullback of the usual Poincaré-Cartan form $\theta_{L}=\frac{\partial L}{\partial \dot{r}^{I}} \mathrm{~d} r^{I}+\frac{\partial L}{\partial \dot{s}^{\alpha}} \mathrm{d} s^{\alpha}$ for $L$ by the injection $i$, i.e. it is a a differential form on $W$ which formally looks similar, but where the coefficients should be evaluated along the constraints. Since $\lambda=i$, the last prolongation bundle $\mu^{i}$ (on which the dynamics $\Gamma$ and the fundamental form $\delta \tilde{\theta}_{L}$ are defined) is the distribution $T^{i} \mathrm{~W}=\mathrm{W} \times{ }_{i} T \mathrm{~W} \rightarrow \mathrm{W}$ of $T \mathrm{~W}$. This distribution, appears also in e.g. [1] (where it is denoted by $H$ ), but its structure as a prolongation bundle was not recognized before. Equations (18) (with $\lambda_{J}^{I}=\delta_{J}^{I}$ and $\lambda_{J}^{\alpha}=-A_{J}^{\alpha}$ ) are of course the required Lagrange-d'Alembert equations

$$
\left\{\begin{array}{l}
\dot{s}^{\alpha}=-A_{I}^{\alpha} \dot{r}^{I}, \\
\frac{\mathrm{d}}{\mathrm{d} t}\left(\frac{\partial L_{c}}{\partial \dot{r}^{I}}\right)=\frac{\partial L_{c}}{\partial r^{I}}-A_{I}^{\alpha} \frac{\partial L_{c}}{\partial s^{\alpha}}-\dot{r}^{J} B_{I J}^{\alpha} \frac{\partial L}{\partial \dot{s}^{\alpha}},
\end{array}\right.
$$

where

$$
B_{I J}^{\alpha}=\frac{\partial A_{I}^{\alpha}}{\partial r^{J}}-\frac{\partial A_{J}^{\alpha}}{\partial r^{I}}+A_{I}^{\beta} \frac{\partial A_{J}^{\alpha}}{\partial s^{\beta}}-A_{J}^{\beta} \frac{\partial A_{I}^{\alpha}}{\partial s^{\beta}} .
$$

The standard way to obtain the equations (19) goes by taking (2) as starting point, and following the same procedure that has led us to (5) in the introduction (see also equations (5.2.7) in [2]). 


\subsection{The reduction of non-holonomic systems with symmetry}

In this example, we will call the configuration space of the mechanical system $Q$ and the Lagrangian $l$. We further assume that $l$ is invariant under the (tangent lift of the) action of a Lie group $G$ and that $\pi_{G}: Q \rightarrow Q / G$ has the structure of a principle fibre bundle. We will first introduce the Lie algebroid structure of interest. It is assumed that the reader is familiar with the natural constructions associated with a principal fibre bundle [11].

Let $\tilde{\mathfrak{g}}=(Q \times \mathfrak{g}) / G \rightarrow Q / G$ be the associated Lie algebra bundle (for a definition see e.g. [13], it is a Lie algebroid structure with vanishing anchor map and structure functions the structure constants of the Lie algebra $\mathfrak{g}$ ). Suppose that $A: T Q \rightarrow \mathfrak{g}$ is a principal connection on $\pi_{G}$ with horizontal distribution $H$. Then $T Q=H \oplus V \pi_{G}$, where $V \pi_{G}=\left\{\left(V \pi_{G}\right)_{q}=T_{q}\left(\pi_{G}^{-1}([q])\right) \mid q \in Q\right\}$ is the vertical distribution. It is shown e.g. in $[6,13]$ that the connection induces a vector bundle isomorphism $\alpha_{A}$ between $T Q / G$ and $T(Q / G) \oplus \tilde{\mathfrak{g}}$ by means of

$$
\alpha_{A}\left(\left[v_{q}\right]\right)=T \pi_{G}\left(v_{q}\right) \oplus\left[q \cdot A\left(v_{q}\right)\right], \quad v_{q} \in T_{q} Q .
$$

Here, $[q \cdot \xi]$ stands for the equivalence class of $(q, \xi) \in Q \times \mathfrak{g}$, i.e. $(q, \xi) \sim\left(q g, A d_{g^{-1}} \cdot \xi\right)$ for all $g \in G$. Remark that $\alpha_{A}(H / G)=T(Q / G)$ and $\alpha_{A}\left(V \pi_{G} / G\right)=\tilde{\mathfrak{g}}$. In the notations from the previous sections, $M=Q / G$. The Lie algebroid is then defined by $\tau: \mathrm{V}=T Q / G \simeq T(Q / G) \oplus \tilde{\mathfrak{g}} \rightarrow Q / G$, where the bracket is taken to be the Lie bracket restricted to right invariant vector fields on $Q$. The anchor map $\rho: \mathrm{V} \rightarrow T M$ of the Lie algebroid is nothing but the projection onto $T(Q / G)$. The Lie algebroid bracket can be given by

$\left[X_{1} \oplus \mathrm{s}_{1}, X_{2} \oplus \mathrm{s}_{2}\right]=\left[X_{1}, X_{2}\right] \oplus\left(\nabla_{X_{1}} \mathrm{~s}_{2}-\nabla_{X_{2}} \mathrm{~s}_{1}-\omega\left(X_{1}, X_{2}\right)+\left[\mathrm{s}_{1}, \mathrm{~s}_{2}\right]\right)$

with $X_{i} \in \mathcal{X}(M), \mathrm{s}_{i} \in \operatorname{Sec}(\tilde{\mathfrak{g}})$. $\nabla$ denotes the covariant derivative on $\tilde{\mathfrak{g}} \rightarrow M$ associated with the connection $A$ and $\omega$ is its curvature.

Recall that a local trivialization of the principal fibre bundle $\pi_{G}$ induces a bundle-adapted coordinate chart on every associated bundle of $\pi_{G}$. In particular, if $e_{\alpha}$ is a basis for the Lie algebra $\mathfrak{g}$, then a basis for the set of local sections of $\tilde{\mathfrak{g}}$ are defined by $\bar{e}_{\alpha}(x)=\left[\psi^{-1}(x, e) \cdot e_{\alpha}\right]$ where $x \in U \subset M=Q / G$ and $\psi: \pi_{G}^{-1}(U) \rightarrow U \times G$ is such a local trivialization of $\pi_{G}$. Suppose $\left(x^{i}, \bar{v}^{\alpha}\right)$ are coordinates on $\tilde{\mathfrak{g}}$ wrt this basis. Then, $\mathrm{V}$ has coordinates $\left(x^{i}, \mathrm{v}^{a}=\left(\dot{x}^{i}, \bar{v}^{\alpha}\right)\right)$. Using this coordinate system, the coefficients of the Lie bracket $\left[\mathrm{s}_{1}, \mathrm{~s}_{2}\right]$ on $\tilde{\mathfrak{g}}$ are exactly the structure constants $C_{\alpha \beta}^{\gamma}$ of $\mathfrak{g}$. Furthermore, the connection coefficients of the covariant derivative $\nabla$ on $\tilde{\mathfrak{g}} \rightarrow Q / G$ associated with $A$ take the form $\Gamma_{i \alpha}^{\beta}=C_{\alpha \gamma}^{\beta} A_{i}^{\gamma}$. Finally, the coefficients of the $\tilde{\mathfrak{g}}$-valued 2-form $\omega$ on $M$ are

$$
\omega_{i j}^{\alpha}=\frac{\partial A_{j}^{\alpha}}{\partial x^{i}}-\frac{\partial A_{i}^{\alpha}}{\partial x^{j}}+C_{\beta \gamma}^{\alpha} A_{j}^{\beta} A_{i}^{\gamma} .
$$

The basis $\left\{\bar{e}_{\alpha}\right\}$ for $\operatorname{Sec}(\tilde{\mathfrak{g}})$ induces a basis $\left\{e_{i}=\frac{\partial}{\partial x^{i}} \oplus 0, e_{\alpha}=0 \oplus \bar{e}_{\alpha}\right\}$ for $\operatorname{Sec}(\tau)$. With respect to this basis, the Lie algebroid bracket (20) is given by

$$
\left[e_{i}, e_{j}\right]=-\omega_{i j}^{\gamma} e_{\gamma}, \quad\left[e_{i}, e_{\alpha}\right]=\Gamma_{i \alpha}^{\gamma} e_{\gamma}, \quad\left[e_{\alpha}, e_{\beta}\right]=C_{\alpha \beta}^{\gamma} e_{\gamma} .
$$

This Lie algebroid structure on $\tau: T Q / G \simeq T M \oplus \tilde{\mathfrak{g}} \rightarrow M$ is the so-called Atiyah algebroid (see also [9, 13]).

Suppose now that the system is subject to some linear constraints $D \subset T Q$. In contrast with the previous example, there is a natural fibration of $Q$ available, $Q \rightarrow M=Q / G$, so it makes sense to compare $D$ with the vertical subspace $V \pi_{G}$ of this fibration, rather than to assume that $D$ is the horizontal distribution of a connection on some fibre bundle. We will follow here the approach of [7], although there are many other [3, 4, 5, 8]. In [7] 
two additional assumptions were made. First, we suppose that $T_{q} Q=D_{q}+\left(V \pi_{G}\right)_{q}$. This assumption means, among others, that $S=\left\{S_{q}=D_{q} \cap\left(V \pi_{G}\right)_{q} \mid q \in Q\right\}$ is a subbundle of $T Q, D$ and $V \pi_{G}$. Further, we will also assume that $D$ (and therefore also $S$ ) is $G$-invariant. In [6] it is proved that there exists always a $G$-invariant metric on $Q$, which we now assume to be fixed. Let $H_{q}$ be the orthogonal complement of $S_{q}$ in $D_{q}$ (with respect to this metric), then $D=H \oplus S$, while $T Q=H \oplus V \pi_{G}$. Further, if $U$ is the orthogonal complement of $S$ in $V \pi_{G}$, then $T Q=H \oplus S \oplus U$. Due to the above assumptions, all three distributions are $G$-invariant and thus $T Q / G=H / G \oplus S / G \oplus U / G$. Let $A: T Q \rightarrow \mathfrak{g}$ be the principal connection whose horizontal subspace at $q$ is exactly $H_{q}$. We now use this connection to consider the above decomposition of $T Q / G$ in the isomorphic bundle $T M \oplus \tilde{\mathfrak{g}}$, i.e. we have

$$
\begin{aligned}
& T M \oplus 0=\alpha_{A}(H / G), \\
& 0 \oplus \tilde{\mathfrak{g}}=\alpha_{A}\left(V \pi_{G} / G\right)=\alpha_{A}(S / G) \oplus \alpha_{A}(U / G)=0 \oplus \tilde{\mathfrak{s}} \oplus \tilde{\mathfrak{u}}, \\
& \alpha_{A}(D / G)=T M \oplus \tilde{\mathfrak{s}} .
\end{aligned}
$$

Analogously to the reduction of non-constrained systems with symmetry, the Lagranged'Alembert principle which describes the equations of motion on $Q$, can be reduced to a 'variational principle' on a reduced space (cf [7]). This new principle generates the reduced equations, the so-called Lagrange-d'Alembert-Poincaré equations. We now show that these equations are Lagrange equations on a subbundle of a Lie algebroid. Using the above notations we now define $\mu: \mathrm{W}=D / G \simeq T M \oplus \tilde{\mathfrak{s}} \rightarrow M$ and this bundle is the required subbundle of the Atiyah algebroid $\tau: \mathrm{V}=T Q / G \simeq T M \oplus \tilde{\mathfrak{g}} \rightarrow M$. Choose a basis $\left\{\bar{e}_{I}\right\}$ of $\operatorname{Sec}(\tilde{\mathfrak{s}})$ and denote $\bar{e}_{I}=e_{I}^{\alpha} \bar{e}_{\alpha}$. Let $\left(x^{i}, \bar{w}^{I}\right)$ be coordinates on $\mathfrak{s}$, then $i: \mathrm{W} \rightarrow \mathrm{V}$ has components $i_{k}^{j}=\delta_{k}^{j}$, $i_{I}^{j}=0, i_{i}^{\alpha}=0$ and $i_{J}^{\alpha}=e_{J}^{\alpha}$. Moreover, the components for $\lambda: \mathrm{W} \rightarrow T M$ are $\lambda_{j}^{i}=\delta_{j}^{i}, \lambda_{J}^{i}=0$.

Let $L \in C^{\infty}(\mathrm{V})$ be the reduced Lagrangian, i.e. $L\left(\left[v_{q}\right]\right)=l\left(v_{q}\right)$. If $L_{c}$ is the restriction of $L$ to $D / G$, then the Lagrange-d'Alembert-Poincaré equations are

$$
\left\{\begin{array}{l}
\frac{\mathrm{d}}{\mathrm{d} t} \frac{\partial L_{c}}{\partial \bar{w}^{I}}=-\frac{\partial L}{\partial \bar{v}^{\beta}}\left(D_{I J}^{\beta} \bar{w}^{J}-\Gamma_{j I}^{\beta} \dot{x}^{j}-\frac{\partial e_{I}^{\beta}}{\partial x^{j}} \dot{x}^{j}\right), \\
\frac{\mathrm{d}}{\mathrm{d} t} \frac{\partial L_{c}}{\partial \dot{x}^{i}}-\frac{\partial L_{c}}{\partial x^{i}}=-\frac{\partial L}{\partial \bar{v}^{\beta}}\left(\Gamma_{i J}^{\beta} \bar{w}^{J}-\omega_{i j}^{\beta} \dot{x}^{j}+\frac{\partial e_{J}^{\beta}}{\partial x^{i}} \bar{w}^{J}\right),
\end{array}\right.
$$

with $D_{I J}^{\beta}=C_{\gamma \delta}^{\beta} e_{I}^{\gamma} e_{J}^{\delta}$ and $\Gamma_{j I}^{\beta}=\Gamma_{j \alpha}^{\beta} e_{I}^{\alpha}$ (see also equations (5.8.45-47) in [2] and (4.11-4.14) in [7]). In $[2,5,7,8]$ one can find examples considered in full detail of non-holonomic systems with symmetry, for instance the snakeboard and the vertically rolling disk.

\subsection{Normal extremals in Lagrangian systems on Lie algebroids}

The last example can be found in the theory of geometric optimal control theory. We first briefly recall some general concepts from control theory. In control theory one studies dynamical systems that can be steered by external devices (typically representing a human input to the system). In dynamical systems theory, such systems are typically represented by a differential equation of the following type:

$$
\dot{x}(t)=f(x(t), u(t)),
$$

where $x \in \mathbb{R}^{n}$ represents the configuration of the system and where $u \in \mathbb{R}^{k}$ represents the control functions. It should be clear that the dynamics of the system is completely determined (up to the initial condition) by the control $u(t)$. A geometric framework for studying control theory is that of an anchored bundle, i.e. we assume that the configuration space is a manifold $M$ and that the control space $\mathbb{R}^{k}$ is the $k$-dimensional fibre of a bundle $\mathrm{V}$ over $M$, with projection $\tau$. 
The analogue of the map $f$ is a bundle map $\rho$ from $\mathrm{V}$ to $T M$, fibred over the identity. An admissible curve is a curve $\mathrm{v}(t)$ in $\mathrm{V}$ such that $\mathrm{d} / \mathrm{d} t(\tau(\mathrm{v}(t)))=\rho(\mathrm{v}(t))$. It is not difficult to see that in a local coordinate system this condition has the precise structure a control differential equation. Assume that a Lagrangian function $L \in C^{\infty}(\mathrm{V})$ is given. In optimal control theory one studies the following variational problem: 'among all admissible curves $\mathrm{v}$ defined over the interval $\left[t_{0}, t_{1}\right]$ and such that $\tau\left(\mathrm{v}\left(t_{0}\right)\right)=x_{0}$ and $\tau\left(\mathrm{v}\left(t_{1}\right)\right)=x_{1}$ for fixed endpoints $x_{0}, x_{1} \in M$, which one minimizes a cost functional $\int L(\mathrm{v}(t)) \mathrm{d} t$ ?'. The maximum principle [20] gives necessary conditions for admissible curves to be minimizing. Locally they are given as follows: an admissible curve $\mathrm{v}(t)=\left(x^{i}(t), \mathrm{v}^{a}(t)\right)$ is minimizing if there is (1) a curve $p_{i}(t)$ in $T^{*} M$ along $x^{i}(t)$ and a constant real number $p_{0}=0,-1$ such that $\left(p_{0}, p_{1}(t), \ldots, p_{n}(t)\right) \neq 0 \forall t$ and (2) the following Hamiltonian system is satisfied at all time $t$, with $H\left(x^{i}, \mathrm{v}^{a}, p_{i}\right)=p_{i} \rho^{i}\left(x^{j}, \mathrm{v}^{a}\right)+p_{0} L\left(x^{i}, \mathrm{v}^{a}\right)$ a function on $T^{*} M \times \mathrm{V}$ :

$$
\begin{aligned}
& \dot{x}^{i}(t)=\frac{\partial H}{\partial p_{i}}=\rho^{i}\left(x^{j}(t), \mathrm{v}^{a}(t)\right), \\
& \dot{p}_{i}(t)=-\frac{\partial H}{\partial x^{i}}=-p_{j} \frac{\partial \rho^{j}}{\partial x^{i}}-p_{0} \frac{\partial L}{\partial x^{i}} \\
& 0=\frac{\partial H}{\partial \mathrm{v}^{a}}=p_{i} \frac{\partial \rho^{i}}{\partial \mathrm{v}^{a}}+p_{0} \frac{\partial L}{\partial \mathrm{v}^{a}} .
\end{aligned}
$$

The latter condition says that the function $\mathrm{v}^{a} \mapsto H\left(x^{i}(t), p_{i}(t), \mathrm{v}^{a}\right)$ on the fibres of $\mathrm{V}$ attains a local maximum at the point $\mathrm{v}^{a}=\mathrm{v}^{a}(t)$. A coordinate free version for this theorem was proven by Sussmann in [23]. However, for the sake of simplicity, we continue to work in a local coordinate system. It is very interesting to note that there are two kinds of solutions: those admissible curves for which there is a $p_{i}(t)$ satisfying the conditions with $p_{0}<0$ and those for which $p_{0}=0$. The latter are called abnormal extremals since in this case the conditions from the maximum principle do not depend on the cost function (see also [12, 18, 19]). If $p_{0}<0$, then the solutions are called normal extremals.

We now assume that the bundle $V$ has the structure of a Lie algebroid, and that the map $\rho$ is the anchor map. We will show that the above conditions from the maximum principle for normal admissible curves can be rewritten as the solutions to Lagrangian systems on Lie algebroids with constraints. For that purpose we consider the equation expressing the maximality condition:

$$
0=\frac{\partial H}{\partial \mathbf{v}^{a}}\left(x^{i}(t), \mathbf{v}^{a}(t), p_{i}(t)\right)=p_{i}(t) \rho_{a}^{i}\left(x^{j}(t)\right)-\frac{\partial L}{\partial \mathbf{v}^{a}}\left(x^{j}(t), \mathrm{v}^{b}(t)\right) .
$$

Since we assumed that the Lagrangian is regular, we can consider the inverse of $\mathbb{F} L=$ $\partial L / \partial \mathrm{v}^{a}: \mathrm{V} \rightarrow \mathrm{V}^{*}$, which gives us the following condition on the control curve:

$$
\mathrm{v}^{a}(t)=\left(\mathbb{F} L^{-1}\right)^{a}\left(x^{j}(t), p_{i}(t) \rho_{a}^{i}\left(x^{j}(t)\right)\right) .
$$

If we substitute this in the Hamiltonian equations from the maximum principle, we obtain after some straightforward calculations (and taking into account the structure equations of the Lie algebroid $\mathrm{V})$ that $\left(x^{i}(t), \mathrm{v}^{a}(t)\right)$ is a solution to the Lagrangian equations on the Lie algebroid:

$$
\left\{\begin{array}{l}
\dot{x}^{i}=\rho_{a}^{i}(x) \mathrm{v}^{a}, \\
\frac{\mathrm{d}}{\mathrm{d} t}\left(\frac{\partial L}{\partial \mathrm{v}^{a}}\right)=\rho_{a}^{i} \frac{\partial L}{\partial x^{i}}-C_{a b}^{c} \mathrm{v}^{b} \frac{\partial L}{\partial \mathrm{v}^{c}} .
\end{array}\right.
$$

This system is in general not equivalent with the Hamiltonian system from the maximum principle. The condition that $\mathrm{v}^{a}$ is in the image of $\mathbb{F} L^{-1}$ has to be taken into account. In the specific case that $\mathbb{F} L^{-1}$ is a linear map, this is precisely saying that $\mathrm{v}(t)$ is contained in 
a linear subbundle $\mathrm{W}$, i.e. $\mathrm{v}(t) \in \mathrm{W}=\operatorname{Im}\left(\mathbb{F} L^{-1} \circ i\left(T^{*} M\right)\right)$. Solutions of the systems (22) and (23) are in fact special solutions of the system (4). So, normal extremals are solutions of a Lagrangian system on a subbundle of a Lie algebroid. However, the equations of motion for the normal extremals are not equivalent to the non-holonomic equations of motion. The normal extremals have to satisfy stronger conditions (23).

A sufficient condition for the image of $\mathbb{F} L^{-1}$ to be a linear subbundle $\mathrm{W}$ of $\mathrm{V}$ is the condition that $L$ is a Lagrangian of mechanical type, i.e. when $L=T-\tau^{*} V$, where $T$ is the kinetic energy associated with a metric on $\mathrm{V}$ and where $V$ is a potential function defined on $M$. A typical example of a mechanical system on a Lie algebroid is the spinning top (cf [14]).

\section{Conclusions and outlook}

In this contribution we have dealt with systems on Lie algebroids that are subject to some constraints. We obtained an intrinsic description of the dynamics of the systems in terms of a section of a prolongation bundle. Our approach unifies models for both Lagrange-d'Alembert equations and Lagrange-d'Alembert-Poincaré equations.

Such a simultaneous description can be very handy in many applications. For example, in [6] it is shown that the so-called 'Lagrange-Poincaré bundles' form the ideal platform on which the reduction process of (unconstrained) Lagrange-Poincaré equations can be repeated. Knowing that such bundles are in fact Lie algebroids, we hope that Lie algebroid theory (and in particular the description of such systems as sections of a prolongation Lie algebroid) will play an important role in future developments of the process called 'Lagrangian reduction by stages'. Due to the observations in this paper, it has become clear that, if we want to find a geometric formalism for successive reduction of non-holonomic systems, we will need to explore the geometry of (the prolongation of ) Lagrange-Poincaré subbundles.

Also in the case of non-holonomic systems (example 3), the above-developed theory leads to interesting new insights. Indeed, the biggest difference between our approach and many others is that the fundamental form $\delta \tilde{\theta}_{L}$ and the dynamics $\Gamma$ are not a differential form or a vector field on $\mathrm{W}$, but a form and a section of the prolongation bundle $T^{i} \mathrm{~W} \rightarrow \mathrm{W}$. A framework that seems to be closely related to ours is [21, 22] (although their set-up is more general since also time-dependent systems were included). In those papers, two fundamental 2 -forms on $T \mathrm{~W}$ have been considered. It would be of interest to explore the relations between those two forms on the one hand and $\delta \tilde{\theta}_{L}$ on the other. Further, we intend to find out how our operator $\delta$ fits in the approach of $[21,22]$.

Another path for future developments is that of an appropriate framework for studying Hamiltonian equations on a subbundle of a Lie algebroid. Hamiltonian systems on Lie algebroids were already considered in e.g. [9, 15]. If $\tau^{*}: \mathrm{V} \rightarrow M$ and $\mu^{*}: \mathrm{W}^{*} \rightarrow M$ are the duals of $\tau$ and $\mu$, then the main object of a Hamiltonian description for constraint systems will be a section of the prolongation bundle $\left(\mu^{*}\right)^{\lambda}: T^{\lambda} \mathbf{W}^{*} \rightarrow \mathrm{W}^{*}$. Similar to before, the, not unrelated, bundles $\left(\tau^{*}\right)^{\rho},\left(\tau^{*}\right)^{\lambda}$ and $\left(\mu^{*}\right)^{\rho}$ will also come into the picture. In the special example of systems with symmetry, we should be able to relate the first equation in $(21)$, in a Hamiltonian formulation, to the momentum equation (for a recent survey see [2]).

\section{Acknowledgments}

We are indebted to Frans Cantrijn, Eduardo Martínez and Willy Sarlet for useful discussions. 


\section{References}

[1] Bates L and Śniatycki J 1992 Nonholonomic reduction Rep. Math. Phys. 32 99-115

[2] Bloch A M 2003 Nonholonomic mechanics and control Interdisciplinary Applied Mathematics vol 24 (New York: Springer)

[3] Bloch A M, Krishnaprasad P S, Marsden J E and Murray R M 1996 Nonholonomic mechanical systems with symmetry Arch. Ration. Mech. Anal. 136 21-99

[4] Cantrijn F, de León M, Marrero J C and Martín de Diego D 1998 Reduction of nonholonomic mechanical systems with symmetries Rep. Math. Phys. 42 25-45

[5] Cantrijn F, de León M, Marrero J C and Martín de Diego D 1999 Reduction of constrained systems with symmetries J. Math. Phys. 40 795-820

[6] Cendra H, Marsden J E and Ratiu T S 2001 Lagrangian Reduction by Stages (Mem. Am. Math. Soc. vol 152)

[7] Cendra H, Marsden J E and Ratiu T S 2001 Geometric mechanics, Lagrangian reduction, and nonholonomic systems Mathematics Unlimited-2001 and Beyond ed B Engquist and W Schmid (Berlin: Springer) pp 221-73

[8] Cortés Monforte J 2002 Geometric, Control and Numerical Aspects of Nonholonomic Systems (Lecture Notes in Math. 1793) (Berlin: Springer)

[9] de León M, Marrero J C and Martínez E 2004 Lagrangian submanifolds and dynamics on Lie algebroids Preprint

[10] Higgins P J and Mackenzie K 1990 Algebraic constructions in the category of Lie algebroids J. Algebra 129 194-230

[11] Kobayashi S and Nomizu K 1963 Foundations of Differential Geometry vols I and II (New York: Intersience)

[12] Langerock B 2003 Geometric aspects of the maximum principle and lifts over a bundle map Acta Appl. Math. 77 71-104

[13] Mackenzie K 1987 Lie Groupoids and Lie algebroids in Differential Geometry (London Math. Soc. Lect. Notes Series vol 124) (Cambridge: Cambridge University Press)

[14] Martínez E 2001 Lagrangian mechanics on Lie algebroids Acta. Appl. Math. 67 295-320

[15] Martínez E 2004 Reduction in optimal control theory Rep. Math. Phys. 53 79-90

[16] Martínez E, Mestdag T and Sarlet W 2002 Lie algebroid structures and Lagrangian systems on affine bundles J. Geom. Phys. 44 70-95

[17] Mestdag T and Sarlet W 2003 The Berwald-type linearisation of generalised connections J. Phys. A: Math. Gen. 36 8049-69

[18] Montgomery R 1994 Abnormal minimizers SIAM J. Control Optim. 32 1605-20

[19] Montgomery R 2002 A Tour of Subriemannian Geometries, their Geodesics and Applications (Mathematical Surveys and Monographs vol 91) (Proludence, RI: American Mathematical Society)

[20] Pontryagin L S, Boltyanskii V G, Gamklelidze R V and Mishchenko E F 1962 The Mathematical Theory of Optimal Processes (New York: Wiley-Interscience)

[21] Sarlet W, Cantrijn F and Saunders D J 1995 A geometrical framework for the study of non-holonomic Lagrangian systems J. Phys. A: Math. Gen. 28 3253-68

[22] Saunders D J, Sarlet W and Cantrijn F 1996 A geometrical framework for the study of non-holonomic Lagrangian systems: II J. Phys. A: Math. Gen. 29 4265-74

[23] Sussmann H J 1997 An introduction to the coordinate-free maximum principle Geometry of Feedback and Optimal Control ed B Jakubczyk and W Respondek (New York: Marcel Dekker) pp 463-557

[24] Weinstein A 1996 Lagrangian mechanics and groupoids Mechanics Day (Waterloo, ON, 1992) Fields Institute Communications vol 7 (Prouidence, RI: American Mathematical Society) pp 207-31 